\title{
Costos de inversión para la descontaminación ambiental mediante modelos econométricos
}

\section{Investment costs for environmental decontamination using econometric models}

\author{
RODRÍGUEZ, Juan P. ${ }^{1}$ \\ RUIZ-OCHOA, Mauricio A. ${ }^{2}$ \\ CASTRO-GARZÓN, Hernando ${ }^{3}$
}

\begin{abstract}
Resumen
Este manuscrito establece elementos económicos para la toma de decisiones en la elaboración de costos en un plan de manejo ambiental, tendientes a reducir de manera significativa los impactos ambientales en los proyectos. Considerando componentes de tratamiento de aguas residuales domésticas, residuos sólidos domésticos, control de la afectación del aire y potabilización del agua como medidas para la reducción de la contaminación, se muestra que las ecuaciones econométricas permiten de manera especial configurar aspectos ambientales más evidentes y eficaces en la descontaminación ambiental.
\end{abstract}

Palabras clave: sostenibilidad, costos de inversión, descontaminación, econometría.

\begin{abstract}
This manuscript establishes economic elements for decision making in the elaboration of costs in an environmental management plan, tending to significantly reduce environmental impacts in projects. Considering components of domestic wastewater treatment, domestic solid waste, air pollution control and water purification as measures for pollution reduction, it is shown that econometric equations allow in a special way to configure more evident and effective environmental aspects in environmental decontamination.
\end{abstract}

Key words: sustainability, investment costs, decontamination, econometrics.

\section{Introducción}

La valoración ambiental de las políticas, programas, proyectos, obras y actividades, conllevan una evaluación del impacto ambiental, evidenciado mediante metodologías específicas según cada contexto y situación propia del proyecto. Esto trae consigo una estrategia articulada en mitigar y reducir los impactos negativos, mediante la elaboración de un plan de manejo encaminado especialmente a la disminución de cada aspecto ambiental valorado dentro una escala de cualificación ambiental, entre bajo y regular. Ello configura una serie de medidas técnicas apropiadas y adecuadas, pero desconociendo el valor de uso directo, indirecto y de existencia, es decir, no siendo preciso en la cuantificación de los costos ambientales de las obligaciones de remediación, cambios en

\footnotetext{
${ }^{1}$ Ingeniero Sanitario y Ambiental. Magister en Ingeniería Ambiental. PhD. en Ingeniería. Profesor Asociado. Universidad Distrital Francisco José de Caldas. Director del grupo de investigación AQUAFORMAT. Correo electrónico: jprodriguezm@udistrital.edu.co.

2 Ingeniero Ambiental. Magister en Ingeniería - Recursos Hidráulicos. Ph.D. en Ingeniería. Docente Investigador. Universidad Manuela Beltrán. Bucaramanga. Colombia.

${ }^{3}$ Profesor, Escuela de Administración y Negocios. Facultad de Ciencias Económicas, Universidad de los Llanos, Villavicencio, Colombia.
} 
la calidad ambiental, agotamiento, impacto estético, emisiones al aire y agua, entre otros (Ruesga \& Durán, 1995; Díaz-Vásquez \& Cancelo, 2010); ello presupone un bajo rigor en lo económico, desconociendo la externalidad económica negativa (perjudicial) el costo de inversión, operación y mantenimiento a nivel de prefactibilidad para la descontaminación y alcanzar los estándares mínimos (Martínez, 1992; Freeman, 1998; Viglizzo et al., 2011), es decir, los valores máximos admisibles establecidos en cada norma local. Por ello, este artículo pretende establecer elementos de ecuaciones econométricas para realizar de una manera eficiente y eficaz la reducción de la contaminación o más bien realizar un seguimiento y control ambiental de la descontaminación desde el ámbito económico.

\section{Metodología}

Con base en las generalidades anteriores, este artículo considera la cuantificación de los costos ambientales de las obligaciones de remediación, y cambios en la calidad ambiental, para ello se presentan aportes relacionados con los Costos de Inversión para Planta Potabilizadoras, Planta de Tratamiento de Agua Residual Municipal (PTARM), Gestión de Residuos Sólidos, y Control de la Contaminación del Aire.

\section{Resultados}

\subsection{Costos ambientales}

Los costos ambientales derivados de la valoración ambiental se pueden establecer de la siguiente forma:

Costos Ambientales = Costos de Gestión Ambiental + Costos por Impacto Ambiental; los Costos de Gestión Ambiental = gestión obligatoria + gestión voluntaria; los costos por impacto ambiental = valoración económica de impactos ambientales (Gómez, 2009; Hincapie \& Becerra, 2014).

Los costos de inversión en el sistema de saneamiento ambiental, según lo establecido por el Banco Mundial y el Ministerio de Desarrollo Económico (Colombia), mencionan que para la captación y desarenación se encuentran entre 4.3 a 15.6 USD\$/habitante; para redes de distribución de agua potable (acueducto) entre 42.8 y 62.7 USD\$/habitante; para tanques de almacenamiento de agua potable entre 17.5 y 32.9 USD\$/habitante; para redes de recolección de aguas residuales domésticas (alcantarillado) entre 108.3 y 196.7 USD\$/habitante; para plantas potabilizadoras entre 10.4 y 25.8 USD\$/habitante; para plantas de tratamiento de agua residuales domésticas entre 35.6 y 48.1 USD\$/habitante.

Según el CEPIS para el año 1999, las tarifas de acueducto y alcantarillado pueden ser: para la Argentina de USD $\$ 0.76 / \mathrm{m}^{3}$ para acueducto y USD $\$ 0.49 / \mathrm{m}^{3}$ para alcantarillado; para Chile de USD $\$ 0.74 / \mathrm{m}^{3}$ para acueducto y USD $\$ 0.36 / \mathrm{m}^{3}$ para alcantarillado; para la Uruguay de USD\$1.81/ $\mathrm{m}^{3}$ para acueducto y USD $\$ 0.82 / \mathrm{m}^{3}$ para alcantarillado; para Perú de USD $\$ 0.57 / \mathrm{m}^{3}$ para acueducto y USD $\$ 0.23 / \mathrm{m}^{3}$ para alcantarillado; para Colombia de USD\$0.57/ $\mathrm{m}^{3}$ para acueducto y USD\$0.41/ $\mathrm{m}^{3}$ para alcantarillado (MDE, 2003).

\section{2. costos de inversión para planta potabilizadoras}

Las ecuaciones econométricas para la estimación de costos de inversión están generalmente establecidas mediante regresión en función de los costos y caudal de la Planta de Tratamiento de Agua Potable (PTAP, a nivel de estudios de factibilidad) mediante la siguiente ecuación exponencial (Pérez, 1992; Valenzuela, 2006; Revollo \& Londoño, 2010; Simate, 2015; León et al., 2016):

$$
C I=a Q^{b},
$$

donde, $C I$ es el costo de inversión o de construcción, $Q$ es el caudal de diseño de la PTAP, $a$ y $b$ son coeficientes calculados. Sin embargo, la constante $a$ representa el costo de capacidad unitaria y la constante $b$ se considera 
como la constante de elasticidad costo escala (siempre positiva). Por lo tanto, si $b=1$ significa que los costos de inversión son directamente proporcionales a la capacidad o caudal de la PTAP (los costos crecen de forma lineal), pero si $b<1$, se entiende que los costos progresan menos que proporcionalmente a la capacidad o tamaño de la PTAP; es decir, se presenta una economía de escala, la cual describe el comportamiento de los costos según la variable del caudal o tamaño (Revollo \& Londoño, 2010). Por otra parte, si el parámetro $b$ es menor en la función de costos de las PTAP, se considera que lentamente crece su costo a medida que se consideran caudales o capacidades mayores de las PTAP; si $b>1$, se presentaría una falsa economía de escala (Valenzuela, 2006). Sin embargo, la literatura reporta valores de la constante $b$ (Cuadro 1) entre 0.68 y 0.954 (Friedler \& Pisanty, 2006; León et al., 2016).

Cuadro 1

Ecuaciones de costos de diversos países.

\begin{tabular}{|c|c|c|c|}
\hline Tecnología & Año & Fuente & Ecuación costo de inversión \\
\hline Filtración rápida convencional & 1977 & CEPIS & $\begin{array}{c}C I=1656 * Q^{0.66} \\
\mathrm{Q}=\text { Caudal }\left(\mathrm{m}^{3} / \mathrm{s}\right) ; \mathrm{Cl}=\text { Costo en miles dólares. }\end{array}$ \\
\hline Filtración rápida modificada & 1977 & CEPIS & $\begin{array}{c}C I=1194 * Q^{0.60} \\
\mathrm{Q}=\text { Caudal }\left(\mathrm{m}^{3} / \mathrm{s}\right) ; \mathrm{Cl}=\text { Costo en miles dólares. }\end{array}$ \\
\hline $\begin{array}{l}\text { Filtración ascendente } \\
\text { descendente }\end{array}$ & 1977 & CEPIS & $\begin{array}{c}C I=1074 * Q^{0.59} \\
\mathrm{Q}=\text { Caudal }\left(\mathrm{m}^{3} / \mathrm{s}\right) ; \mathrm{Cl}=\text { Costo en miles dólares. }\end{array}$ \\
\hline Filtración ascendente & 1977 & CEPIS & $\begin{array}{c}C I=950 * Q^{0.59} \\
\mathrm{Q}=\text { Caudal }\left(\mathrm{m}^{3} / \mathrm{s}\right) ; \mathrm{Cl}=\text { Costo en miles dólares. }\end{array}$ \\
\hline Sistema convencional & 2010 & ACP (Panamá) & $C I=1 \mathrm{USD} \$=1 \mathrm{Gal} / \mathrm{día}$ \\
\hline
\end{tabular}

En Colombia, se han realizado varios análisis de regresión de exponencial, donde se han formulado funciones de costos para diversas tecnologías de PTAP (Cuadro 2).

\section{Cuadro 2}

Ecuaciones de costos para Colombia.

\begin{tabular}{|c|c|c|c|}
\hline Tecnología & Año & Fuente & Ecuación costo de inversión \\
\hline Sistema convencional & 1982 & $\begin{array}{l}\text { Instituto Nacional de } \\
\text { Fomento Municipal } \\
\text { (INSFOPAL) }\end{array}$ & $\begin{aligned} & C I=2078 * Q^{0.53}(1 \leq \mathrm{Q} \leq 150 \mathrm{~L} / \mathrm{s}) \\
Q= & \text { Caudal }(\mathrm{L} / \mathrm{s}) ; C I=\text { Costo en miles dólares }\end{aligned}$ \\
\hline Sistema convencional & 1982 & INSFOPAL & $\begin{array}{c}C I=437 * Q^{0.83}(1 \leq \mathrm{Q} \leq 150 \mathrm{~L} / \mathrm{s}) \\
Q=\text { Caudal }(\mathrm{L} / \mathrm{s}) ; C I=\text { Costo en miles dólares }\end{array}$ \\
\hline Sistema convencional & 1998 & MDE (2003) & $\begin{array}{c}C I=70,52 * Q^{0.36}(2 \leq \mathrm{Q} \leq 100 \mathrm{~L} / \mathrm{s}) \\
Q=\text { Caudal }(\mathrm{L} / \mathrm{s}) ; C I=\text { Costo en miles COLPESOS }\end{array}$ \\
\hline Filtración directa & 1998 & MDE (2003) & $\begin{array}{c}C I=62.68 * Q^{0.32}(2 \leq \mathrm{Q} \leq 100 \mathrm{~L} / \mathrm{s}) \\
Q=\text { Caudal }(\mathrm{L} / \mathrm{s}) ; C I=\text { Costo en miles COLPESOS }\end{array}$ \\
\hline Filtración múltiple etapas & 1998 & MDE (2003) & $\begin{array}{c}C I=38.60 * Q^{0.67} \quad(2 \leq \mathrm{Q} \leq 100 \mathrm{~L} / \mathrm{s}) \\
Q=\text { Caudal }(\mathrm{L} / \mathrm{s}) ; C I=\text { Costo en miles COL PESOS }\end{array}$ \\
\hline Filtración gruesa y directa & 1998 & MDE (2003) & $\begin{array}{c}C I=38.60 * Q^{0.67}(2 \leq \mathrm{Q} \leq 100 \mathrm{~L} / \mathrm{s}) \\
Q=\text { Caudal }(\mathrm{L} / \mathrm{s}) ; C I=\text { Costo en miles COLPESOS }\end{array}$ \\
\hline Sistema convencional & 2001 & $\begin{array}{c}\text { Díaz-Granados et al. } \\
(2002)\end{array}$ & $\begin{array}{c}C I=13,010 * Q^{0.8082} \\
Q=\text { Caudal }(\mathrm{L} / \mathrm{s}) ; C I=\text { Costo en miles COL PESOS }\end{array}$ \\
\hline $\begin{array}{l}\text { Sistema convencional } \\
\text { (Compacta - Fibra de vidrio) }\end{array}$ & 2001 & $\begin{array}{c}\text { Díaz-Granados et al. } \\
(2002)\end{array}$ & $\begin{array}{c}C I=21,886 * Q^{0.5734} \\
Q=\text { Caudal }(\mathrm{L} / \mathrm{s}) ; C I=\text { Costo en miles COLPESOS }\end{array}$ \\
\hline Sistema convencional & 2005 & $\begin{array}{c}\text { Ministerio de } \\
\text { Ambiente, Vivienda y } \\
\text { Desarrollo Territorial } \\
\text { (MAVDT) }\end{array}$ & $\begin{array}{c}C I=91 * Q^{0.588} \\
Q=\text { Caudal }(\mathrm{L} / \mathrm{s}) ; C I=\text { Costo en miles COLPESOS }\end{array}$ \\
\hline $\begin{array}{l}\text { Sistema convencional } \\
\text { (Compacta - Fibra de vidrio) }\end{array}$ & 2006 & Rodríguez et al. (2015) & $\begin{array}{c}C I=81,231,292.53 * Q^{2.837}(0.5 \leq Q \leq 1.5 \mathrm{~L} / \mathrm{s}) \\
Q=\text { Caudal }(\mathrm{L} / \mathrm{s}) ; C I=\text { Costo en COLPESOS }\end{array}$ \\
\hline
\end{tabular}




\begin{tabular}{|c|c|c|c|}
\hline Tecnología & Año & Fuente & $\begin{array}{l}\text { Ecuación costo de inversión } \\
\end{array}$ \\
\hline & & & $\begin{array}{c}C I=31,467,947.5 * Q^{2.536}(1.5 \leq \mathrm{Q} \leq 3.0 \mathrm{~L} / \mathrm{s}) \\
Q=\text { Caudal }(\mathrm{L} / \mathrm{s}) ; C I=\text { Costo en } \operatorname{COLPESOS} \\
C I=125,155.7 * Q^{5.182}(4.0 \leq \mathrm{Q} \leq 6.0 \mathrm{~L} / \mathrm{s}) \\
Q=\text { Caudal }(\mathrm{L} / \mathrm{s}) ; C l=\text { Costo en } \mathrm{COL} \mathrm{PESOS}\end{array}$ \\
\hline Sistema convencional & 2014 & $\begin{array}{l}\text { León et al. (2016); } \\
\text { Rodríguez et al. (2015) }\end{array}$ & $\begin{array}{c}C I=2 \times 10^{8} * Q^{0.5442}(5 \leq \mathrm{Q} \leq 80 \mathrm{~L} / \mathrm{s}) \\
Q=\text { Caudal }(\mathrm{L} / \mathrm{s}) ; C l=\text { Costo en COL PESOS }\end{array}$ \\
\hline $\begin{array}{l}\text { Sistema convencional } \\
\text { (Compacta - Fibra de vidrio) }\end{array}$ & 2014 & $\begin{array}{l}\text { León et al. (2016); } \\
\text { Rodríguez et al. (2015) }\end{array}$ & $\begin{array}{c}C I=1 \times 10^{8} * Q^{0.8056}(0.1 \leq \mathrm{Q} \leq 5 \mathrm{~L} / \mathrm{s}) \\
Q=\text { Caudal }(\mathrm{L} / \mathrm{s}) ; C I=\text { Costo en COL PESOS }\end{array}$ \\
\hline $\begin{array}{l}\text { Sistema convencional } \\
\text { (Semicompacta - Fibra de } \\
\text { Vidrio) }\end{array}$ & 2015 & León et al. (2016) & $\begin{array}{c}C I=1 \times 10^{7} * Q+2 \times 10^{7}(0.3 \leq \mathrm{Q} \leq 10 \mathrm{~L} / \mathrm{s}) \\
Q=\text { Caudal }(\mathrm{L} / \mathrm{s}) ; C I=\text { Costo en COLPESOS }\end{array}$ \\
\hline
\end{tabular}

Fuente: León et al. (2016)

La ecuación de costo de capacidad (Blank \& Tarquin, 1992; Peters et al., 2003), asumiendo que el valor $x=0.44$ (sistemas de saneamiento) se tiene:

$$
C I_{2014 Q 20}=C I_{2014 Q 5} *\left(\frac{Q_{\mathrm{i}+1}}{Q_{\mathrm{i}}}\right)^{x}
$$

donde, $Q_{i+1}$ es la capacidad instalada requerida (L/s), $Q_{i}$ es la capacidad instalada actual (L/s), y $x$ es un coeficiente de mayoración.

\subsection{Costos de inversión para Planta de Tratamiento de Agua Residual Municipal (PTARM)}

El costo directo de construcción o de inversión aproximados a nivel de prefactibilidad para la asignación eficiente de recursos (Onkal \& Demir, 2006) y la medición de los impactos de los usuarios finales de un proyecto de PTARM, indican el orden de prioridad de la inversión, y comparan entre varios proyectos de PTARM, sin incluir aspectos como la localización, área, impactos ambientales y los precios locales en el momento de la construcción, entre algunos aspectos. Ciertas regresiones de exponenciales se han diferenciado según el nivel o proceso de tratamiento de las aguas residuales, como se muestra en el Cuadro 3:

Cuadro 3

Ecuaciones de costos de diversos países.

\begin{tabular}{|c|c|c|c|c|c|}
\hline Tecnología & $\begin{array}{l}\text { Función de costos } \\
\text { (CI) }\end{array}$ & $\begin{array}{l}\text { No. de } \\
\text { datos }\end{array}$ & $\begin{array}{c}\text { Límite de } \\
\text { confiabiabilidad } \\
(\mathrm{L} / \mathrm{s})\end{array}$ & $\begin{array}{c}\text { Coeficiente } \\
\text { de } \\
\text { correlación }\end{array}$ & Fuente \\
\hline $\begin{array}{l}\text { Tratamiento } \\
\text { secundario }\end{array}$ & $C I=8988 * Q^{0.71}$ & 37 & $16.20-1388.9$ & 0.908 & $\begin{array}{l}\text { Friedler \& Pisanty } \\
(2006)\end{array}$ \\
\hline $\begin{array}{l}\text { Secundario avanzado } \\
\text { y nitrificación }\end{array}$ & $C I=2790 * Q^{0.84}$ & 11 & $34.7-173.6$ & 0.938 & $\begin{array}{l}\text { Friedler \& Pisanty } \\
(2006)\end{array}$ \\
\hline Lodos activados & $\begin{array}{l}C I \\
=0.0031 * Q^{0.881}\end{array}$ & 6 & $115.7-289.3$ & 0.979 & $\begin{array}{l}\text { Singhirunnusorn \& } \\
\text { Stenstrom (2018) }\end{array}$ \\
\hline Zanjón de oxidación & $\begin{array}{l}\text { CI } \\
=0.0017 * Q^{0.910}\end{array}$ & 8 & $11.6-902.8$ & 0.604 & $\begin{array}{l}\text { Singhirunnusorn \& } \\
\text { Stenstrom (2018) }\end{array}$ \\
\hline Lagunas aireadas & $\begin{array}{l}C I \\
=0.0143 * Q^{0.681}\end{array}$ & 11 & $11.6-902.8$ & 0.822 & $\begin{array}{l}\text { Singhirunnusorn \& } \\
\text { Stenstrom (2018) }\end{array}$ \\
\hline Lagunas de oxidación & $\begin{array}{l}C I \\
=0.0004 * Q^{1.060}\end{array}$ & 23 & $11.6-902.8$ & 0.790 & $\begin{array}{l}\text { Singhirunnusorn \& } \\
\text { Stenstrom (2018) }\end{array}$ \\
\hline $\begin{array}{l}\text { Tratamiento } \\
\text { secundario } \\
\text { convencional }\end{array}$ & $C I=0.116 * Q^{0.854}$ & 9 & NA & 0.935 & $\begin{array}{l}\text { Tsagarakis et al. } \\
\text { (2003) }\end{array}$ \\
\hline $\begin{array}{l}\text { Aireación extendida } \\
\text { mecánica }\end{array}$ & $C I=0.206 * Q^{0.775}$ & 35 & NA & 0.829 & $\begin{array}{l}\text { Tsagarakis et al. } \\
(2003)\end{array}$ \\
\hline
\end{tabular}




\begin{tabular}{|c|c|c|c|c|c|}
\hline Tecnología & $\begin{array}{l}\text { Función de costos } \\
\text { (CI) }\end{array}$ & $\begin{array}{l}\text { No. de } \\
\text { datos }\end{array}$ & $\begin{array}{c}\text { Límite de } \\
\text { confiabiabilidad } \\
\text { (L/s) }\end{array}$ & $\begin{array}{l}\text { Coeficiente } \\
\text { de } \\
\text { correlación }\end{array}$ & Fuente \\
\hline $\begin{array}{l}\text { Aireación extendida } \\
\text { aire disuelto }\end{array}$ & $C I=0.153 * Q^{0.727}$ & 32 & NA & 0.808 & $\begin{array}{l}\text { Tsagarakis et al. } \\
\text { (2003) }\end{array}$ \\
\hline Tratamiento primario & $C I=15.75 * Q^{0.684}$ & NA & $1-4,000$ & 1.000 & Aristizábal (2011) \\
\hline $\begin{array}{l}\text { Tratamiento } \\
\text { secundario }\end{array}$ & $C I=23.46 * Q^{0.763}$ & NA & $1-5,000$ & 1.000 & Aristizábal (2011) \\
\hline
\end{tabular}

Cuadro 4

Ecuaciones de costos de diversos países.

\begin{tabular}{|c|c|c|c|c|c|}
\hline Tecnología & Función de costos & $\begin{array}{l}\text { No. de } \\
\text { datos }\end{array}$ & $\begin{array}{c}\text { Límite de } \\
\text { confiabilidad (L/s) }\end{array}$ & $\begin{array}{l}\text { Coeficiente } \\
\text { de } \\
\text { correlación }\end{array}$ & Fuente \\
\hline $\begin{array}{l}\text { Lagunas de } \\
\text { estabilización }\end{array}$ & $C I=41915593 * Q^{0.4019}$ & 7 & $1-250$ & NA & $\begin{array}{l}\text { Buitrago \& } \\
\text { Giraldo (1994) }\end{array}$ \\
\hline UASB & $C I=13974805 * Q^{0.8149}$ & 5 & $1-450$ & NA & $\begin{array}{l}\text { Buitrago \& } \\
\text { Giraldo (1994) }\end{array}$ \\
\hline RAP & $C I=43108293 * Q^{0.4243}$ & 2 & $1-60$ & NA & $\begin{array}{l}\text { Buitrago \& } \\
\text { Giraldo (1994) }\end{array}$ \\
\hline $\begin{array}{l}\text { Aireación } \\
\text { extendida }\end{array}$ & $C I=33826482 * Q^{0.8378}$ & 3 & $1-40$ & NA & $\begin{array}{l}\text { Buitrago \& } \\
\text { Giraldo (1994) }\end{array}$ \\
\hline $\begin{array}{l}\text { Tratamiento } \\
\text { secundario }\end{array}$ & $\begin{array}{c}C I=2841 * Q^{2}+46830 * Q \\
-18.34\end{array}$ & NA & $1000-14000$ & 0,984 & $\begin{array}{l}\text { Aristizábal } \\
\text { (2011) }\end{array}$ \\
\hline $\begin{array}{l}\text { Reactor } \\
\text { Anaerobio }\end{array}$ & $\begin{array}{rl}C I=248.263 .192 & * Q^{0.88} \\
& * D B O^{0.53} \\
& * N^{0.11} * S S T^{0.30} \\
& * P^{0.03}\end{array}$ & 30 & $1-250$ & 0.985 & $\begin{array}{l}\text { Rodríguez et } \\
\text { al. (2015) }\end{array}$ \\
\hline $\begin{array}{l}\text { Lagunas de } \\
\text { Oxidación }\end{array}$ & $\begin{aligned} & C I=2,501,108,824 * Q^{0.59} \\
& * D B O^{0.28} \\
& * N^{0.65} * S S T^{0.06} \\
& * P^{0.93}\end{aligned}$ & 30 & $1-250$ & 0.985 & $\begin{array}{l}\text { Rodríguez et } \\
\text { al. (2015) }\end{array}$ \\
\hline $\begin{array}{l}\text { Reactor de } \\
\text { Lodos } \\
\text { Activados }\end{array}$ & $\begin{array}{rl}C I=1,672,784 & * Q^{0.33} * D B O^{2.18} \\
& * N^{0.18} * S S T^{0.96} \\
& * P^{0.32}\end{array}$ & 30 & $1-250$ & 0.985 & $\begin{array}{l}\text { Rodríguez et } \\
\text { al. (2015) }\end{array}$ \\
\hline
\end{tabular}

$C I$ en millones de dólares y $Q$ en $\mathrm{m}^{3} / \mathrm{s}$. Fuente: León et al. (2016)

\subsection{Costos de inversión para la gestión de residuos sólidos}

Los costos de inversión en el sistema de aseo (residuos sólidos) según lo establecido por el Banco Mundial y el Ministerio de Desarrollo Económico (Colombia), son: para rellenos sanitarios convencionales de 3.0 USD\$/habitantes y para la recolección de residuos sólidos urbanos de 5.5 USD\$\$/habitante. Collazos \& Duque (1988) establece que el costo de inversión en relleno sanitario para el año 2002 es de USD\$0.63 por $\mathrm{m}^{3}$ depositado; en el año 2000, el costo de inversión estaba entre 35 a 100 USD\$/tonelada dispuesta. La función de costos de inversión es de $C I=4318.08 * T^{0.514}$ y los costos de operación y mantenimiento $C I=4318.08 *$ $T^{0.514}$ (Collazos \& Duque, 1988). 
De otro lado, los costos para la inversión instalada para rellenos sanitarios en Latinoamérica y el Caribe oscilan entre 5,000 a USD\$15,000/tonelada. Los costos de inversión para compostaje, entre 20,000 a USD\$ 40.000/tonelada. Los costos de inversión para incineración entre 125,000 a USD\$160,000/tonelada (Jaramillo, 2003).

Adicionalmente, los costos de inversión para planta de reciclaje oscilan entre 45 a 150 USD\$/tonelada recuperada. Los costos de inversión para una planta de lixiviados oscilan entre 10 a $40 \mathrm{USD} \$ / \mathrm{m}^{3}$ tratado. Un equipo de digestión anaerobia para residuos sólidos tiene un costo de inversión entre 15.5 y 17 USD\$ millones para 192 y 200 toneladas/día, y para la operación de entre 11.14 y 19.52 USD\$/tonelada. Para los equipos de incineración, los costos de inversión entre 300 a 400 USD\$ millones para una capacidad de 1,200 toneladas/día (es decir 500 a 1,000 USD\$/tonelada); los costos de operación entre 100 a 150 USD\$/tonelada (Solórzano \& Villalba, 2018). Los costos de inversión según Terraza \& Willumsen (2010) para plantas de aprovechamiento de biogás de alto BTU oscilan entre 1,800 a 4,000 USD\$ por $\mathrm{m}^{3}$ /día, y la evaporación de lixiviado oscila entre 7,000 a 10,000 USD\$ por $\mathrm{m}^{3}$ /día (Cervi et al., 2011)

Los costos de operación del sistema de aseo, en Latinoamérica: la recolección puede estar entre 30 a 80 USD\$/ton, transferencia entre 10 a 20USD\$/ton, y disposición final 10 a 20 USD\$/ton; los costos de operación de disposición final en Estados Unidos (USA) 30 USD\$/ton, incineración de 60 USD\$/ton; en Europa el costo promedio de operación en la disposición final es de 40 a 60 USD\$/ton; en Colombia, en el Relleno Sanitario Doña Juana (Bogotá) el costo de operación es de 6.6 USD\$/ton y el costo total de servicio de aseo (disposición final, barrido/limpieza, recolección y transporte) es 12.41 USD\$/ton (Cervi et al., 2011; Hincapie \& Becerra, 2014; Solórzano \& Villalba, 2018).

\subsection{Costos de inversión para el control de la contaminación del aire}

Los costos de inversión (capital total) para la reducción de la contaminación del aire según lo menciona Bravo (2000) y EPA (2002) handbrook air pollution control cost manual, para precipitadores electroestáticos $C_{I}=$ $1502739 * Q^{0.564160}$, y los costos de operación son:

$$
C_{O}=(66.11 * Q+805817) * H R S P,
$$

donde, $Q$ es el caudal de la fuente emisora, y HRSP son las horas promedio de operación al día.

Para filtros de mangas es $C_{I}=827728.102 * Q^{0.403027}$;

Para los costos de operación es $C_{O}=(1643.998 * Q+9381097) * H R S P$;

Para lavador venturi el costo de inversión es $C_{I}=1391 * Q^{0.98}$;

y el costo de operación $C_{O}=(1533.4 * Q+288916) * H R S P$ (Freeman, 1998; Feal, 2002; Mejía \& Oviedo, 2006; Manzano-Agugliaro \& Carrillo-Valle, 2016).

Para ciclones, de flujo de 0,5 a $12 \mathrm{~m}^{3} / \mathrm{s}$; temperatura máxima del gas de entrada $540^{\circ} \mathrm{C}$; carga de MP de 2.3 a 230 $\mathrm{g} / \mathrm{m}^{3}$ y eficiencia del $90 \%$. $C_{I}$ (1995) 4600 a USD $\$ 7400$ por m³ $/ \mathrm{s}$. Para flujo de 12 a $50 \mathrm{~m}^{3} / \mathrm{s}$. $C_{I}$ (1995) de 4100 a USD $\$ 5,000$ por $\mathrm{m}^{3} / \mathrm{s}$. Adicionalmente, para filtros de mangas de flujo de 1 a $470 \mathrm{~m}^{3} / \mathrm{s}$; temperatura hasta $260^{\circ} \mathrm{C}$; Carga de 9 a $120 \mathrm{~g} / \mathrm{m}^{3}$. $C_{I}$ (1995) 13,000 a USD $\$ 55,000$ por $\mathrm{m}^{3} / \mathrm{s}$. Los costos de operación y mantenimiento, para Ciclones convencionales (2002) entre 4600 a USD $\$ 7400$ por $\mathrm{m}^{3} / \mathrm{s}$; Ciclones múltiples (1995) entre USD $\$ 1,600$ a USD $\$ 2,600$ por $\mathrm{m}^{3} / \mathrm{s}$; para Filtros de Mangas (2002) entre 11,000 a USD\$50,000 por m³/s (Wark, 1996; EPA, 2002; Evans, 2004). 


\section{Conclusiones}

Los costos de inversión y/ de capital evidencian una aproximación a nivel de prefactibilidad en proyectos de descontaminación, mediante planes de manejo ambiental. Se muestran diversas tecnologías en el tratamiento de aguas residuales, potabilización, control de la contaminación del aire y residuos sólidos, que se pueden ajustar en diversos contextos y especialmente condiciones de proyectos que ameritan una evaluación económica contigua, no solo en aspectos de la inversión sino también de la operación y mantenimiento de los sistemas propuestos.

\section{Referencias bibliográficas}

Aristizábal, O.L. (2011). Referencias para costos de inversión en plantas de tratamiento de aguas residuales. En: Pinilla, J.I. Las ciudades y el agua: Ingenieros de EPM investigan sobre los sistemas hídricos urbanos. Medellín: Universidad de Medellín.

Blank, L. \& Tarquin, A. (1992). Ingeniería económica. Texas, USA: McGraw Hill.

Buitrago, C.A. \& Giraldo, E. (1994). Evaluación técnica y económica de sistemas de tratamiento de aguas residuales. Bogotá: Universidad de los Andes.

Bravo, R. (2000). Proposición y Evaluación de Instrumentos de incentivo Económico para Mejorar la Calidad del Aire en Santiago: Aplicación al Caso de Fuentes Fijas. Tesis de Ingeniero Civil, Facultad de Ciencias Físicas y Matemáticas, Universidad de Chile.

Cervi, R.; Esperancini, M. \& Bueno, O. (2011). Viabilidad Económica de la Utilización de Biogás para la Conversión en Energía Eléctrica. Información Tecnológica, 22(4): 3-14.

Collazos, H. \& Duque, R. (1988). Residuos Sólidos. Bogotá: FUNPIRS.

Díaz-Granados, M.; Barrera, S.; Ramos, J.; Camacho, L.; Rosales, R.; Escalante, N. \& Torres, M. (2002). Metodología multicriterio para la priorización de inversión de aguas residuales municipales en Colombia. XX Congreso Latinoamericano de Hidráulica.

Díaz-Vázquez, M.R. \& Cancelo, M.T. (2010). Análisis de los factores determinantes de la evolución de las emisiones de $\mathrm{CO}_{2}$ y de azufre en países OCDE mediante una descomposición econométrica. Revista De Economía Mundial, 26: 85-106.

EPA. (2002). Manual de costos de control de contaminación del aire de la EPA. USA: U.S. Environmental Protection Agency, USEPA.

Evans, R. (2004). Peer Review of BenMAP Software. USA: Environmental Protection Agency.

Feal, A. (2002). Filtros de mangas (I). Características típicas y mecanismos básicos. Ingeniería química, 34(39): 458-469.

Freeman, H. (1998). Manual de prevención de la contaminación industrial. Mexico: McGraw-Hill.

Friedler, E. \& Pisanty, E. (2006). Effects of design flow and treatment level on construction and operation costs of municipal wastewater treatment plants and their implications on policy making. Water Research, 40(20): 3751-3758.

Gómez, M. (2009). Tensiones, posibilidades y riesgos de la contabilidad medioambiental empresarial. Contaduría Universidad de Antioquia, 54: 55-78. 
Hincapie, D. \& Becerra, W.L. (2014). Gestión de costos ambientales hacia el desarrollo sostenible. Propuesta para su valoración y revelación contable. Trabajos de grado Contaduría Pública, 8(1).

Jaramillo, J. (2003). Centro Panamericano de Ingeniería Sanitaria y Ciencias del Ambiente; Efectos de la inadecuada gestión de Residuos sólidos. Universidad de Antioquía, Medellín.

León, K.; Rey, G.L. \& Rodríguez, J.P. (2016). Ecuaciones econométricas para los costos de inversión en plantas de tratamiento de agua potable en Colombia. I+D Revista de investigaciones, 7(1): 109 - 115.

Manzano-Agugliaro, F. \& Carrillo-Valle, J. (2016). Conversion of an existing electrostatic precipitator casing to Pulse Jet Fabric filter in fossil power plants. Revista Dyna, 83(195): 189-197.

Martínez, J. (1992). Evaluación costo beneficio del control de la contaminación del aire en el área metropolitana de Monterrey. Nuevo León: Universidad de Autónoma de Nuevo León.

Mejía, M.E. \& Oviedo, I.D. (2006). Estimación de las funciones de costo marginal de abatimiento de material particulado para fuentes fijas en el Valle de Aburrá. Ensayos de Economía, 16(29): 55-81.

Ministerio de Desarrollo Económico (MDE). (2003). Sector de agua potable y saneamiento básico: retos y resultados. Bogotá: Ministerio de Desarrollo Económico. Colombia.

Onkal G. \& Demir, I. (2006). Cost analysis of alternative methods for wastewater handling in small communities. Journal of Environmental Management, 79(4): 357-363.

Pérez Carrión, J. (1992). Mejoramiento de calidad del agua para consumo humano. Lima: OPS/CEPIS.

Peters, M.; Timmerhaus, K. \& West, R. (2003). Plant design and economics for chemical engineers. Firth edition. USA: Mc Graw-Hill.

Revollo D. \& Londoño, G. (2010). Análisis de las economías de escala y alcance en los servicios de acueducto y alcantarillado en Colombia. Desarrollo y sociedad, 66: 145- 182.

Rodríguez, J.P. (2009). Selección técnico económico del sistema de depuración de aguas residuales. Aplicando la evaluación de la descontaminación hídrica. Tecnología del Agua, 29(306): 22-31.

Rodríguez, J.P.; García-Ubaqué, C. \& Penagos-Londoño, J.C. (2015). Analysis of the investment costs in municipal wastewater treatment plants in Cundinamarca. Revista Dyna, 82(192): 230-238.

Ruesga, S.M. \& Durán, G. (1995). Empresa y medio ambiente. Madrid: Pirámide.

Simate, G. (2015). The treatment of brewery wastewater for reuse by integration of coagulation/flocculation and sedimentation with carbon nanotubes 'sandwiched' in a granular filter bed. Journal of Industrial and Engineering Chemistry, 21: 1277-1285.

Singhirunnusorn, W. \& Stenstrom, M. (2010). A critical analysis of economic factors for diverse wastewater treatment processes: Case studies in Thailand. Sustainable Environment Research, 20: 263-268.

Solórzano, G. \& Villalba, L. (2018). Capitulo V. Tratamiento y valorización de residuos sólidos urbanos. En: Tello, P.; Campani, D. \& Sarafian, D. Gestión Integral de residuo sólidos urbanos. México: AIDIS.

Terraza, H. \& Willumsen, H. (2010). Guidance Note on Landfill Gas Capture and Utilization. Inter - American Development Bank. 68 p. 
Tsagarakis, K.P.; Mara, D.D. \& Angelakis, A.N. (2003). Application of cost criteria for selection of municipal wastewater treatment systems. Water, air and soil pollution, 142: 187-210.

Valenzuela L.C. (2006). Evaluación económica y metodología de minimización de costos para proyectos de sistemas de agua potable. Desarrollo y sociedad, 19: 147- 171.

Viglizzo, E.; Carreño, L.; Volante, J. \& Mosciaro, M.J. (2011). Valuación de los bienes y servicios ecosistémicos: Verdad objetiva o cuento de la buena pipa? En: Laterra, P.; Jobbagy, E. \& Paruelo, J.M. Valoración de Servicios Ecosistémicos. Conceptos, herramientas y aplicaciones para el ordenamiento territorial, Chapter: 1, Publisher: Ediciones INTA. pp. 19-25.

Wark, K. (1996). Contaminación del aire. Origen y control. México: Limusa. 